\title{
Investigation of MEK activity in COS7 cells entering mitosis
}

\author{
HUAIPING SHI, TIANYING ZHANG, YONGQING YI and JUN LUO \\ College of Animal Science and Technology, Northwest A\&F University, Yangling, Shaanxi 712100, P.R. China
}

Received December 3, 2013; Accepted May 19, 2014

DOI: $10.3892 / \mathrm{mmr} .2014 .2590$

\begin{abstract}
Although the mitogen-activated protein kinase (MAPK) pathway has been extensively investigated, numerous events remain unclear. In the present study, we examined mitogen-activated protein kinase kinase (MEK) expression from interphase to mitosis. Following nocodazole treatment, COS7 cells gradually became round as early as $4 \mathrm{~h}$ after treatment. Cyclin B1 expression gradually increased from 4 to $24 \mathrm{~h}$ in the presence of nocodazole. When cells were treated with nocodazole for $4 \mathrm{~h}$, the level of epidermal growth factor (EGF)-mediated MEK phosphorylation did not significantly change between nocodazole-untreated and -treated $(4 \mathrm{~h})$ cells $(\mathrm{P}>0.05)$. However, EGF-mediated MEK phosphorylation was significantly inhibited upon treatment with nocodazole for 8 and $24 \mathrm{~h}$ compared to nocodazole-untreated cells $(\mathrm{P}<0.05)$. MEK phosphorylation levels were comparable between $1,5,10$ and $50 \mathrm{ng} / \mathrm{ml}$ EGF treatments. Phorbol 12-myristic 13-acetate (PMA) did not activate MEK in mitotic cells. Following treatment of COS7 cells at the interphase with AG1478 or U0126, MEK phosphorylation was blocked. In addition, the investigation of the expression of proteins downstream of MEK demonstrated that EGF does not significantly affect the phosphorylation level of extracellular-signal-regulated kinase (ERK), ribosomal protein S6 kinase (RSK) and Elk in mitotic cells $(\mathrm{P}>0.05)$. The results showed that MEK expression is gradually inhibited from cell interphase to mitosis, and that MEK downstream signaling is affected by this inhibition, which probably reflects the requirements of cell physiology during mitosis.
\end{abstract}

\section{Introduction}

Mitogen-activated protein kinase kinase (MEK), also known as MAP2K, is a kinase enzyme that phosphorylates mitogen-activated protein kinases, for example the mitogen-activated protein kinase (MAPK), or the extracellular-signal-regulated kinase (ERK). When MEK is inhibited, cell proliferation is

Correspondence to: Dr Huaiping Shi, College of Animal Science and Technology, Northwest A\&F University, 22 Xinong Road, Yangling, Shaanxi 712100, P.R. China

E-mail:shp1974yuan@sina.com

Key words: MEK, EGF, mitosis blocked and apoptosis (controlled cell death) is induced. The small-molecule MEK inhibitor trametinib displayed substantial clinical activity in melanoma (1), which suggests that MEK is a valid therapeutic target.

The cell cycle is a series of events that takes place in a cell, leading to its division and replication. The cell cycle is divided into two brief periods in eukaryotes: the interphase and the mitotic phase. At the interphase, distinct signaling events in the Ras-ERK pathway facilitate the progression through G1/S and survival in G1, via nuclear transcription factor phosphorylation, immediate-early gene induction, expression of cell cycle genes that direct DNA synthesis, and regulation of translational initiation $(2,3)$. However, a number of studies on cell mitosis have shown that protein traffic through the membrane is inhibited, and the transmembrane signals emitted by mitotic cells are likely blocked (4-8). For example, the epidermal growth factor receptor (EGFR) is hyperphosphorylated in cell mitosis, which decreases its binding affinity to EGF (9).Eventually, EGF-mediated signaling is attenuated (9-12). The Ras-ERK pathway, one of the EGF-mediated signaling pathways, has been extensively investigated for its important physiological roles in the cell cycle (13-15). This pathway comprises a number of important signaling proteins such as Ras, Raf, MEK and ERK. Raf is phosphorylated by Ras, activated Raf phosphorylates and activates MEK, which in turn, phosphorylates and activates ERK. Previous reports have demonstrated that MEK activity is inhibited in mitosis (16-18). The $\mathrm{N}$ terminal of phosphorylated MEK1 was shown to be hydrolyzed in mitosis, which attenuated its binding to ERK, and eventually to blocking of the related signaling transduction pathways. However, MEK was also proposed to be activated by phorbol 12-myristic 13-acetate (PMA) in mitosis (11). Therefore, MEK activity in mitosis still remains unclear.

In this study, we analyzed MEK activity in response to nocodazole in COS7 cells from the interphase to mitosis. MEK phosphorylation gradually decreased with prolonged treatment with nocodazole. The phosphorylation of the MEK downstream proteins ERK, ribosomal protein S6 kinase (RSK) and Elk was also inhibited by nocodazole in cell mitosis.

\section{Materials and methods}

Cells lines and culture. $\operatorname{COS} 7$ cells were grown at $37^{\circ} \mathrm{C}$ in Dulbecco's modified Eagle's medium (DMEM) containing $10 \%$ fetal bovine serum (FBS), penicillin and streptomycin, and were maintained in a $5 \% \mathrm{CO}_{2}$ atmosphere. 
Antibodies and chemicals. Mouse anti-tubulin and -phosphorylated (p)p-ERK, rabbit anti-MEK1/2, -p-RSK, -p-Elk and -cyclin B1, goat anti-p-EGFR (1086) and -p-MEK1/2 were purchased from Santa Cruz Biotechnology, Inc. (Santa Cruz, CA, USA). Horseradish peroxidase (HRP)-conjugated secondary antibodies and nitrocellulose membranes were purchased from Bio-Rad (Hercules, CA, USA). EGF was obtained from Upstate Biotechnology, Inc. (Lake Placid, NY, USA). Nocodazole, AG1478, U0126 and PMA were from Calbiochem ${ }^{\circledast}$ (Merck KGaA, Darmstadt, Germany). DMEM was from Gibco ${ }^{\circledR}$ (Grand Island, NY, USA). Unless otherwise specified, all chemicals were purchased from Sigma-Aldrich (St. Louis, MO, USA).

Cell morphology in response to nocodazole. COS7 cells at $80 \%$ confluence were treated with $0.25 \mu \mathrm{g} / \mathrm{ml}$ nocodazole for $0,4,8,24 \mathrm{~h}$, and their morphology was observed under an inverted microscope (Nikon Diaphot; Nikon, Tokyo, Japan). Images of COS7 cells were acquired at x10 magnification.

EGF-mediated MEK activity in nocodazole-treated cells. COS7 cells were first treated with or without $250 \mathrm{ng} / \mathrm{ml}$ nocodazole for different time periods $(0,4,8,24 \mathrm{~h})$, and then stimulated with $50 \mathrm{ng} / \mathrm{ml}$ EGF for $15 \mathrm{~min}$. Finally, all cells were collected and lysed to analyze MEK phosphorylation.

MEK activity in different concentrations of EGF. Asynchronous cells were stimulated by treatment with 50, 10, 5 and $1 \mathrm{ng} / \mathrm{ml} \mathrm{EGF}$ for $15 \mathrm{~min}$, followed by cell lysis prior to analysis of MEK phosphorylation.

MEK activity in cell cycle. Following treatment with or without nocodazole, COS7 cells were stimulated by treatment with EGF or PMA for different time periods $(0,5,15 \mathrm{~min})$. Subsequently, asynchronous and mitotic cells were collected and lysed to analyze the phosphorylation of EGFR and MEK.

MEK activity in response to protein inhibitors. Following treatment of COS7 cells with $0.5 \mu \mathrm{M}$ AG1478 for $30 \mathrm{~min}$ or $10 \mu \mathrm{M} \mathrm{U} 0126$ for $60 \mathrm{~min}$, the cells were stimulated with $50 \mathrm{ng} / \mathrm{ml} \mathrm{EGF}$ for $15 \mathrm{~min}$, followed by cell lysis prior to analysis of MEK phosphorylation.

Activity of MEK downstream proteins. Following overnight treatment with nocodazole, mitotic COS7 cells were stimulated by $50 \mathrm{ng} / \mathrm{ml}$ EGF for $0,15,30$ and $60 \mathrm{~min}$, followed by detection of ERK, RSK and Elk phosphorylation levels and data analysis.

Western blotting. Cells were lysed with radioimmunoprecipitation assay buffer (50 mM Tris-HCl, $\mathrm{pH} 8.0 ; 150 \mathrm{mM} \mathrm{NaCl}$, $1 \%$ Nonidet P-40, $1 \%$ sodium deoxycholate and $0.1 \%$ SDS) supplemented with protease inhibitor cocktail (Roche Inc., Basel, Switzerland). Cell lysates were separated by electrophoresis on $7.5-10 \%$ sodium dodecyl sulfate polyacrylamide gels, depending on the size of the studied proteins. Prestained protein markers were used as molecular weight standards. Proteins were electrophoretically transferred onto nitrocellulose membranes. Blots were blocked with 3\% skim milk in $0.05 \%$ Tween-phosphate buffered saline for $30 \mathrm{~min}$.

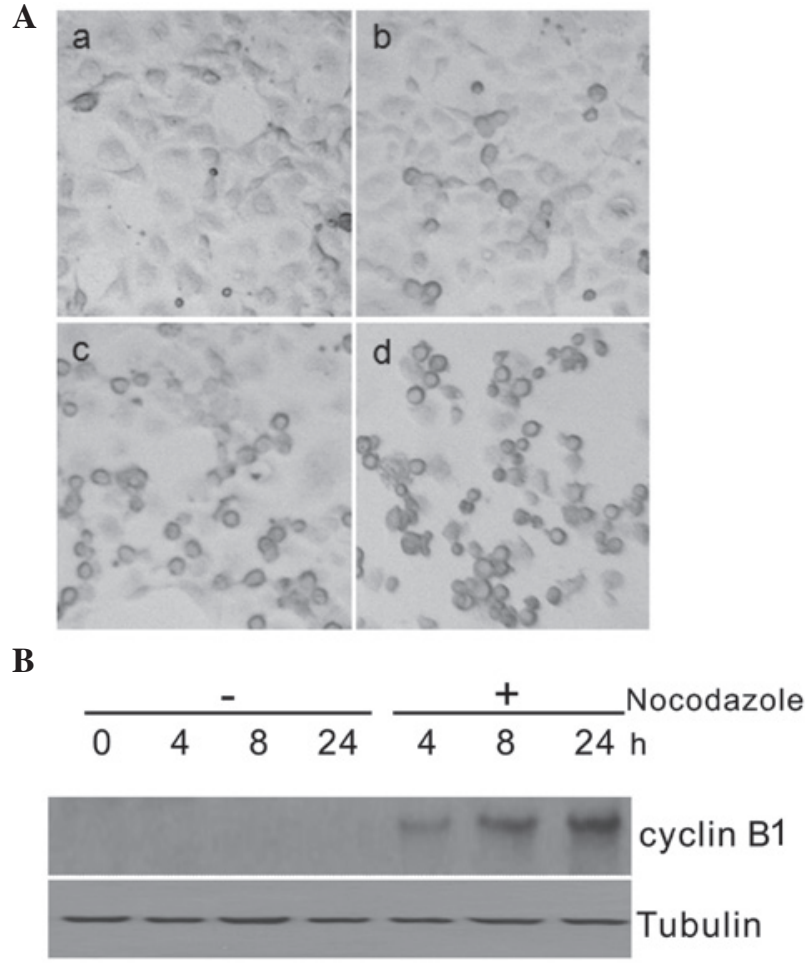

Figure 1. (A) COS7 cells observed under the microscope following treatment with or without nocodazole for (a) 0, (b) 4, (c) 8 and (d) 24 h. (B) Western blot showing cyclin B1 expression in the cells after treatment. Tubulin was used as the loading control.

Membranes were then incubated overnight with monoclonal mouse anti-tubulin and -pp-ERK, polyclonal rabbit anti-MEK1/2, -p-RSK, -p-Elk and -cyclin B1, polyclonal goat anti-p-EGFR (1086), and -p-MEK1/2 in blocking buffer. The membranes were then incubated with a polyclonal rabbit anti-goat or a goat anti-mouse IgG coupled to HRP for $1 \mathrm{~h}$, and the primary antibodies were detected using enhanced chemiluminescence (ECL), using Pierce ${ }^{\mathrm{TM}}$ SuperSignal ECL Western Blotting Detection reagents (Pierce Chemical Co., Rockford, IL, USA). Light detection was performed with a Fuji photo film (Tokyo, Japan).

Statistical analysis. Three independent experiments were performed in all cases. The data from western blots were expressed as means $\pm \mathrm{SE}$ of triplicate measurements, and were analyzed with the software package SPSS 16.0 (SPSS Inc., Chicago, IL, USA). In data graphs, different letters among bars indicate a significant difference $(\mathrm{P}<0.05)$ between values.

\section{Results}

Cell morphology. Following treatment with nocodazole, the COS7 cell morphology changed. At $4 \mathrm{~h}$, a few cells became round. At $8 \mathrm{~h}$, more cells were round. Up to $24 \mathrm{~h}$, almost all of cells had become round, which suggests that cells distinctly enter and stay in mitosis (Fig. 1A). Cell morphology changes indicated that cellular signaling may be gradually altered. Therefore, the expression of cyclin B1, which is a marker of mitosis, was investigated by western blotting. The results demonstrated that the expression of cyclin B1 prominently changed upon nocodazole treatment (Fig. 1B). 
A

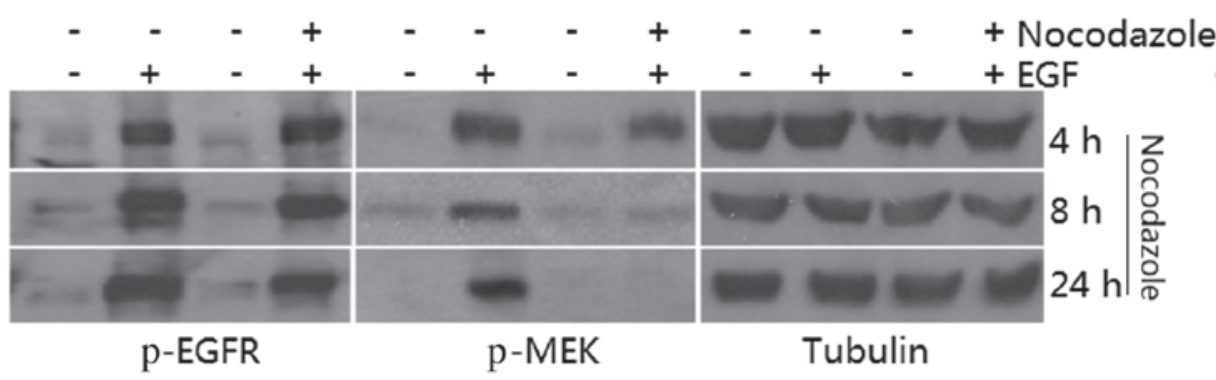

B
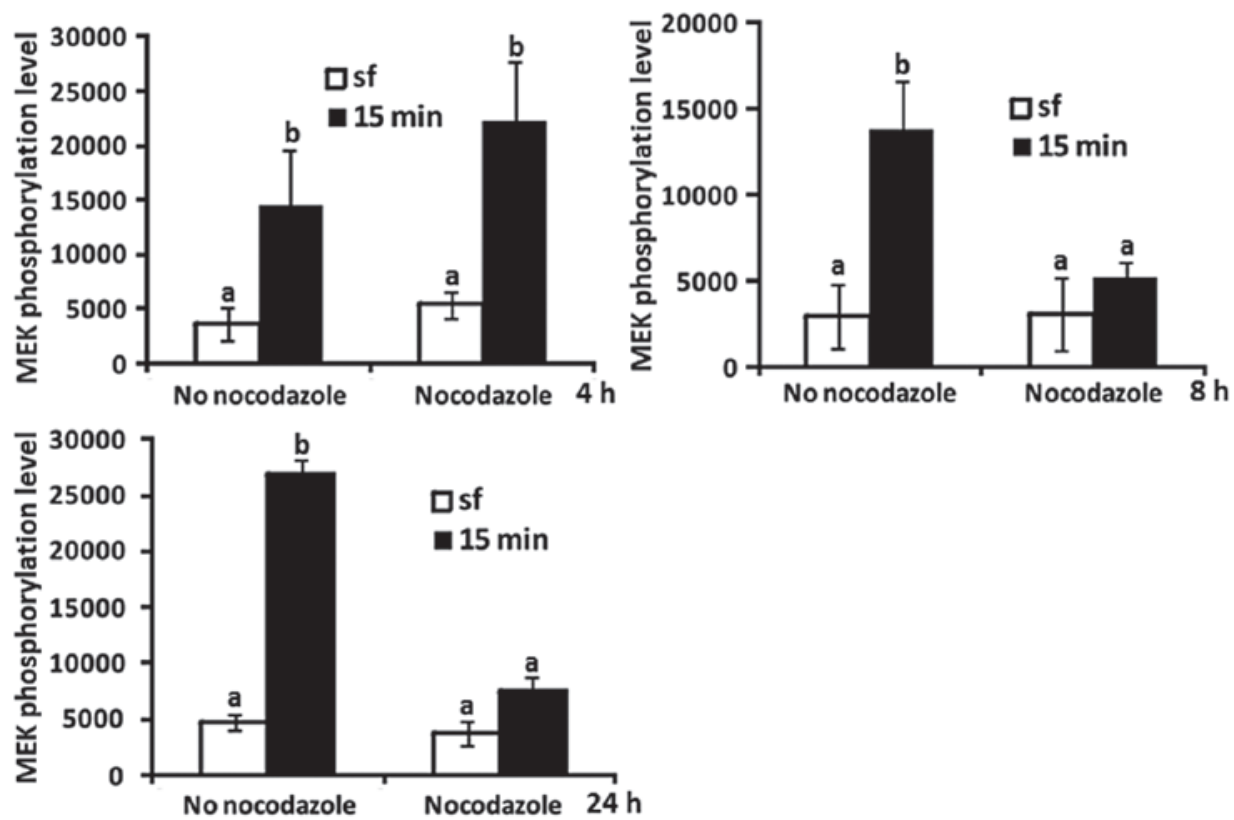

Figure 2. Effects of COS7 cell treatment with or without EGF $(50 \mathrm{ng} / \mathrm{ml})$ for $15 \mathrm{~min}$ following treatment with or without nocodazole $(250 \mathrm{ng} / \mathrm{ml})$ for 4,8 and $24 \mathrm{~h}$. (A) The levels of phosphorylated EGFR and MEK (p-EGFR and p-MEK) were evaluated by immunoblotting. Tubulin was used as the loading control. (B) Quantified data of MEK phosphorylation from three independent experiments. EGFR, epidermal growth factor receptor; MEK, mitogen-activated protein kinase kinase; sf, serum free.

In nocodazole-untreated cells, cyclin B1 was nearly undetectable from 0 to $24 \mathrm{~h}$. By contrast, cyclin B1 was detected in nocodazole-treated cells, and its level gradually increased from 4 to $24 \mathrm{~h}$, which indicates that an increasing number of COS7 cells entered and were subsequently arrested in mitosis.

MEK phosphorylation in the transition of cell phases. MEK is an important signaling protein of the MAPK pathway. Following treatment with nocodazole for 4,8 and $24 \mathrm{~h}, \operatorname{COS} 7$ cells were treated with EGF for $15 \mathrm{~min}$, and were collected to investigate MEK phosphorylation. The p-EGFR level was increased when cells were stimulated by EGF in both nocodazole-treated and untreated cells (Fig. 2A), and no difference in p-EGFR levels was observed betweeen these cells. These results indicated that EGFR is activated during the cell cycle, which possibly initiates the MAPK pathway. When cells were treated with nocodazole for $4 \mathrm{~h}$ and EGF for $15 \mathrm{~min}$, MEK was significantly phosphorylated in nocodazole-treated cells, with the p-MEK level being similar to that of nocodazole-untreated cells (Fig. 2B). In COS7 cells treated with nocodazole for longer periods, MEK phosphorylation was significantly $(\mathrm{P}<0.05)$ inhibited after 15 min of EGF stimulation, especially following a 24-h nocodazole treatment (Fig. 2B). These results indicate that MEK phosphorylation is blocked from interphase to mitosis.

$M E K$ phosphorylation in response to EGF. Although EGF-mediated MEK phosphorylation was inhibited when cells entered mitosis, the effects of different EGF doses on this inhibition remained unclear. The level of p-MEK was revealed to be similar among asynchronous cells treated with different EGF concentrations (50, 10, 5 and $1 \mathrm{ng} / \mathrm{ml}$ ) (Fig. 3A), which indicates that the dose of EGF does not affect MEK phosphorylation. In addition, we collected mitotic cells and detected EGFR expression by western blotting. EGFR phosphorylation was induced by EGF, but not by PMA, alone or in combination with nocodazole treatment (Fig. 3B). By contrast, MEK was not phosphorylated by EGF or PMA in the presence of nocodazole (Fig. 3B). These results indicated the presence of a yet unidentified mechanism inhibiting MEK phosphorylation in mitosis.

MEK phosphorylation and the roles of inhibitors. Two important inhibitors of signaling proteins were employed to treat COS7 cells. AG1478, a specific inhibitor of EGFR, was used to block EGFR phosphorylation. Following treatment with $0.5 \mu \mathrm{M}$ 


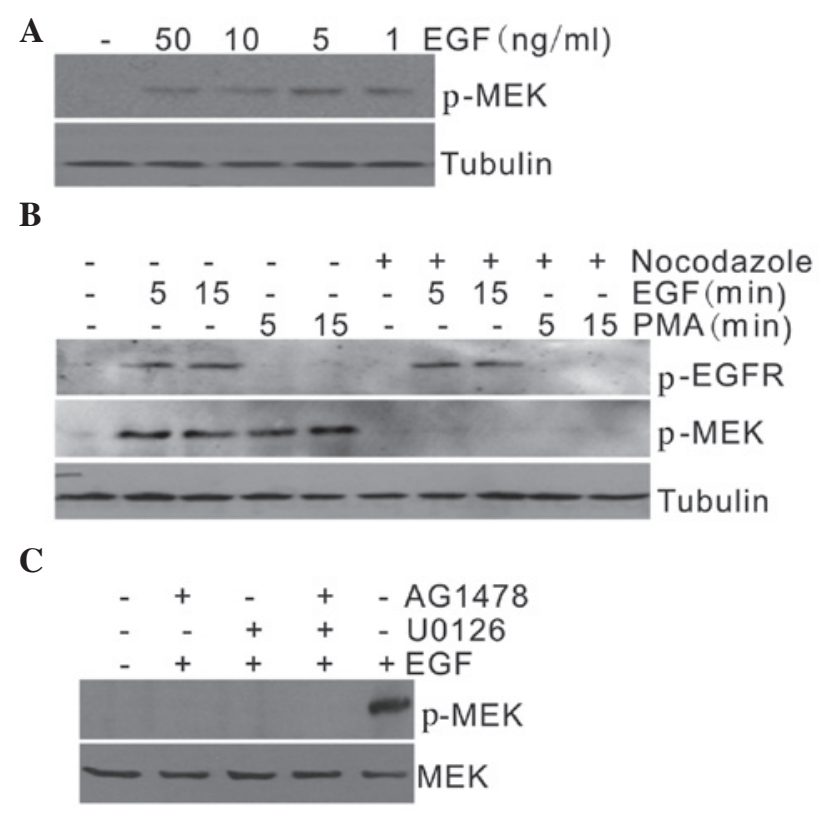

Figure 3. (A) MEK phosphorylation in COS7 cells at the interphase following treatment with or without different concentrations of EGF $(50,10,5,1 \mathrm{ng} / \mathrm{ml})$ for 15 min. (B) Phosphorylation of EGFR and MEK in asynchronous and mitotic COS7 cells, collected after overnight treatment with or without nocodazole, followed by stimulation with EGF and PMA. (C) MEK phosphorylation in COS7 cells at the interphase after treatment with AG1478 and U0126. Tubulin was used as the loading control in all experiments. MEK, mitogen-activated protein kinase kinase; EGFR, epidermal growth factor receptor; PMA, phorbol myristic acid; p, phosphorylated.

AG1478 for $30 \mathrm{~min}, \mathrm{COS} 7$ cells were treated with $50 \mathrm{ng} / \mathrm{ml}$ EGF for $15 \mathrm{~min}$. The results demonstrated that MEK phosphorylation was decreased (Fig. 3C), which suggests that EGFR activity is important for MEK phosphorylation. Because AG1478 only indirectly affects MEK phosphorylation, U0126, which is a specific MEK inhibitor, was used to treat COS7 cells. Following treatment with $10 \mu \mathrm{M}$ U0126 for $60 \mathrm{~min}, \mathrm{COS} 7$ cells were treated with $50 \mathrm{ng} / \mathrm{ml}$ EGF for $15 \mathrm{~min}$. EGF-mediated MEK phosphorylation was again reduced (Fig. 3C), which confirmed the ability of U0126 to inhibit MEK phosphorylation.

MEK signaling in mitosis. Since MEK phosphorylation was impaired in mitotic cells, the phosphorylation of the downstream signaling proteins ERK, RSK and Elk was next investigated. ERK is a downstream protein of MEK, and RSK is a downstream protein of ERK. Elk is a transcription activator which also acts downstream of ERK. Following overnight treatment with nocodazole, COS7 cells were treated with EGF. Elk phosphorylation was increased by nocodazole treatment compared to the control $(\mathrm{P}<0.05$, Fig. 4C). However, stimulation of COS7 cells with EGF during 15, 30 and $60 \mathrm{~min}$ did not significantly enhance the phosphorylation levels of ERK, RSK and Elk in the mitotic cells $(P>0.05$, Fig. 4). While RSK phosphorylation dropped after 30 min of EGF stimulation (Fig. 4B), Elk and ERK phosphorylation steadily increased with prolonged EGF treatment in mitotic cells (Fig. 4A and C).

\section{Discussion}

In the present study, we demonstrated that $\operatorname{COS} 7$ cells are gradually arrested in mitosis in response to nocodazole. MEK

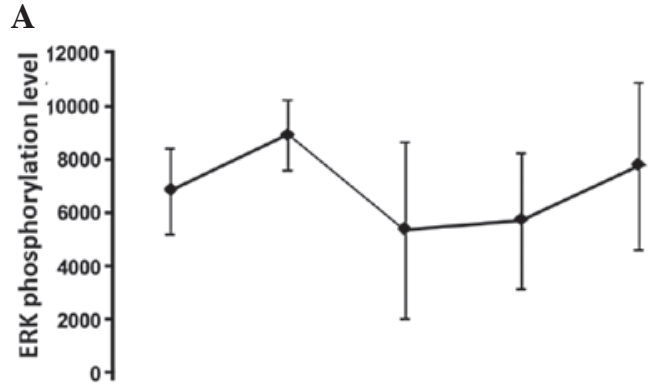

B

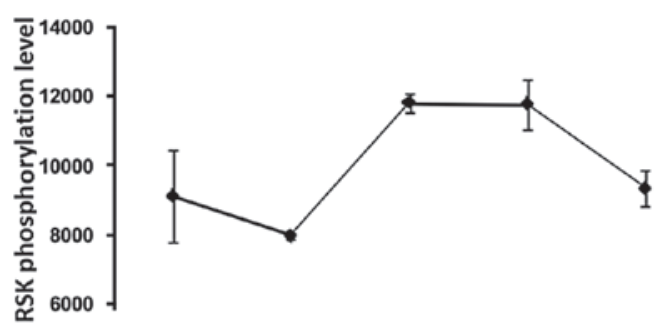

C

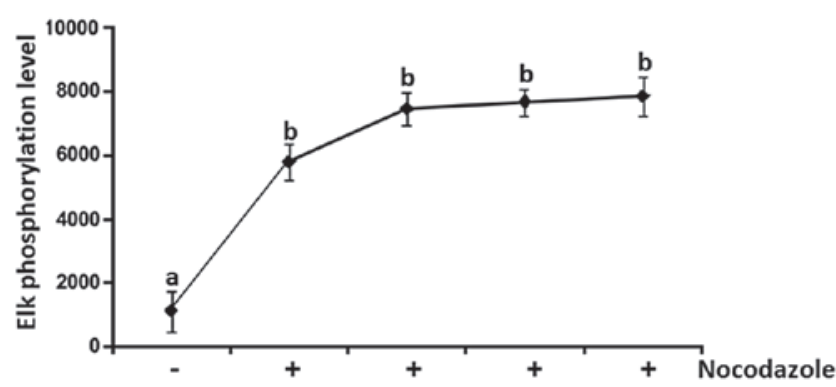

Figure 4. Phosphorylation of (A) ERK, (B) RSK and (C) Elk in COS7 mitotic cells after overnight treatment with nocodazole, followed by stimulation with EGF for 15, 30 and 60 min. EGF, epidermal growth factor; ERK, extracellular-signal-regulated kinase; RSK, ribosomal protein S6 kinase.

activity was also gradually inhibited in these conditions. At 24 h, MEK phosphorylation was significantly inhibited by nocodazole compared to the control. Nocodazole treatment significantly increased the phosphorylation of the MEK downstream protein Elk, while an increase in ERK phosphorylation was also observed. However, RSK phosphorylation was decreased by nocodazole treatment, and the phosphorylation levels of all three proteins were not significantly $(\mathrm{P}>0.05)$ increased in the presence of EGF in the mitotic cells. These results suggest that the activity of MEK protein is blocked in response to nocodazole treatment.

An increasing number of studies has provided evidence that cellular innate molecular events change at the transition from the interphase to mitosis $(10,19,20)$. The MAPK pathway has been shown to be affected by changes in the regulation and control of signaling proteins during mitosis $(16,21)$. Here, we used COS7 cells as experimental models to analyze MEK activity from the interphase to mitosis.

As previously reported, cells became round in the presence of nocodazole (22). Cell morphology was gradually altered: After nocodazole treatment for $4 \mathrm{~h}$, a few cells reacted and their shape was round. More cells were round after 
treatment with nocodazole for $24 \mathrm{~h}$. Moreover, the expression of cyclin B1, a marker of mitosis, in nocodazole-treated cells was increased with prolonged nocodazole treatment compared to nocodazole-untreated cells, which further supports that COS7 cells are arrested in the mitosis and are a suitable model for the study of changes in cell-cycle proteins.

To analyze MEK activity from interphase to mitosis, we collected all cells, including interphase and mitotic cells, after nocodazole treatment. Regarding the activation of EGFR, we found that EGFR is phosphorylated in COS7 cells following EGF treatment. EGFR phosphorylation levels were not different between nocodazole-treated and -untreated cells from 4 to $24 \mathrm{~h}$, which indicates that EGFR phosphorylation is required in the cell cycle. EGFR activation initiates downstream signaling cascades, with a number of signaling proteins potentially activated (23-26). Based on this, we next detected MEK activation from interphase to mitosis using western blotting. We demonstrated that MEK is significantly phosphorylated upon EGF treatment in cells treated with nocodazole for $4 \mathrm{~h}(\mathrm{P}<0.05)$. Nevertheless, after COS7 cells were treated with nocodazole for 8 and up to $24 \mathrm{~h}$, MEK phosphorylation was significantly inhibited compared to nocodazole-untreated cells $(\mathrm{P}<0.05)$. These results probably relate to the number of mitotic cells. Treatment with nocodazole for $4 \mathrm{~h}$ induced mitotic arrest in a few cells, while most of the cells remained at the interphase. Since MEK phosphorylation was increased at the interphase, MEK might still have remained activated by EGF in cells treated with nocodazole for $4 \mathrm{~h}$. However, after $8 \mathrm{~h}$ of treatment with nocodazole, more cells were arrested in mitosis, which is expected to decrease MEK phosphorylation. In any case, nocodazole treatment appeared to affect the cell cycle and related proteins. Therefore, we conclude that MEK phosphorylation is significantly and gradually blocked by prolonged nocodazole treatment.

Although MEK activity was shown to be stimulated by EGF, the effect of the EGF dose remained unclear. We thus collected cells and detected the effect of the EGF dose on MEK phosphorylation. MEK phosphorylation remained unchanged in the presence of different concentrations of EGF, not exceeding $50 \mathrm{ng} / \mathrm{ml}$. We therefore selected the concentration of $50 \mathrm{ng} / \mathrm{ml} \mathrm{EGF}$ for the following experiments. After cells were treated with nocodazole and EGF, EGFR was activated in asynchronous and mitotic cells, which possibly initiated the downstream signaling pathways. MEK was activated in nocodazole-untreated cells but inhibited in nocodazole-treated cells, which indicates that MAPK might be blocked by nocodazole in mitosis. To further confirm this, we treated the COS7 cells with PMA. MEK phosphorylation was not increased with PMA treatment in mitotic cells. A previous study (11) demonstrated that MEK is activated by PMA-activated protein kinase C (PKC). Since MEK was not activated by EGF and PMA in mitotic cells, we conclude that an unknown signaling pathway is blocked in mitosis, and thereby MEK phosphorylation is downregulated.

Signaling transduction is blocked by protein inhibitors (27). To further investigate MEK phosphorylation in mitosis, AG1478, an EGFR inhibitor, was used to treat COS7 cells. The results demonstrated that AG1478 inhibits MEK phosphorylation, which indicates that EGFR activity is important for the activation of MEK. Moreover, MEK phosphorylation was inhibited by U0126, a highly selective inhibitor of both MEK1 and MEK2 $(28,29)$. In addition, AG1478 and U0126 displayed a synergistic effect on MEK phosphorylation. These results indicate that there are numerous factors affecting MEK activity.

Extracellular-signal-regulated kinases (ERKs) are widely expressed intracellular signalling kinases that are involved in functions such as regulation of meiosis and mitosis, and post-mitotic functions in differentiated cells. In the MAPK/ERK pathway, Ras activates c-Raf, followed by MEK and then ERK1/2. ERKs are known to activate numerous transcription factors, such as RSK and Elk1 $(30,31)$. By investigating the expression of downstream proteins of MEK, we found that the phosphorylation of RSK is reduced in mitotic cells upon nocodazole treatment. Although ELK appeared to be activated by nocodazole $(\mathrm{P}<0.05)$, Elk phosphorylation was not significantly increased by EGF treatment in mitotic cells $(\mathrm{P}>0.05)$. These results indicate that the activity of $\mathrm{MEK}$ is downregulated in mitosis.

Overall, our study demonstrated that MEK activity is gradually blocked from cell interphase to mitosis, which probably follows the energy requirements needed for the dynamic structural changes occurring in mitosis. Nevertheless, certain molecular events require further investigation.

\section{Acknowledgements}

This study was jointly supported funding from the Northwest A\&F University (grant no. QN2011010) and the National Natural Science Foundation of China (grant no. 31272409).

\section{References}

1. Falchook GS, Lewis KD, Infante JR, et al: Activity of the oral MEK inhibitor trametinib in patients with advanced melanoma: a phase 1 dose-escalation trial. Lancet Oncol 13: 782-789, 2012.

2. Lavoie JN, L'Allemain G, Brunet A, Muller R and Pouyssegur J: Cyclin D1 expression is regulated positively by the $\mathrm{p} 42 / \mathrm{p} 44 \mathrm{MAPK}$ and negatively by the p38/HOGMAPK pathway. J Biol Chem 271: 20608-20616, 1996.

3. Weber JD, Raben DM, Phillips PJ and Baldassare JJ: Sustained activation of extracellular-signal-regulated kinase 1 (ERK1) is required for the continued expression of cyclin D1 in G1 phase. Biochem J 326: 61-68, 1997.

4. Kreiner T and Moore HP: Membrane traffic between secretory compartments is differentially affected during mitosis. Cell Regul 1: 415-424, 1990.

5. Berlin RD, Oliver JM and Walter RJ: Surface functions during mitosis I: phagocytosis, pinocytosis and mobility of surface-bound Con A. Cell 15: 327-341, 1978.

6. Berlin RD and Oliver JM: Surface functions during mitosis. II. Quantitation of pinocytosis and kinetic characterization of the mitotic cycle with a new fluorescence technique. J Cell Biol 85: 660-671, 1980.

7. Sager PR, Brown PA and Berlin RD: Analysis of transferrin recycling in mitotic and interphase HeLa cells by quantitative fluorescence microscopy. Cell 39: 275-282, 1984.

8. Warren G, Featherstone C, Griffiths G and Burke B: Newly synthesized $G$ protein of vesicular stomatitis virus is not transported to the cell surface during mitosis. J Cell Biol 97: $1623-1628,1983$

9. Kiyokawa N, Lee EK, Karunagaran D, Lin SY and Hung MC: Mitosis-specific negative regulation of epidermal growth factor receptor, triggered by a decrease in ligand binding and dimerization, can be overcome by overexpression of receptor. J Biol Chem 272: 18656-18665, 1997.

10. Gomez-Cambronero J: p42-MAP kinase is activated in EGF-stimulated interphase but not in metaphase-arrested HeLa cells. FEBS Lett 443: 126-130, 1999. 
11. Klein S, Kaszkin M, Barth H and Kinzel V: Signal transduction through epidermal growth factor receptor is altered in HeLa monolayer cells during mitosis. Biochem J 322: 937-946, 1997.

12. Newberry EP and Pike LJ: Cell-cycle-dependent modulation of EGF-receptor-mediated signaling. Biochem Biophys Res Commun 208: 253-259, 1995.

13. Choi $\mathrm{C}$ and Helfman DM: The Ras-ERK pathway modulates cytoskeleton organization, cell motility and lung metastasis signature genes in MDA-MB-231 LM2. Oncogene: Sep 2, 2013 (Epub ahead of print)

14. Cha DS, Datla US, Hollis SE, Kimble J and Lee MH: The Ras-ERK MAPK regulatory network controls dedifferentiation in Caenorhabditis elegans germline. Biochim Biophys Acta 1823: 1847-1855, 2012.

15. Yoon $\mathrm{S}$ and Seger R: The extracellular signal-regulated kinase: multiple substrates regulate diverse cellular functions. Growth Factors 24: 21-44, 2006.

16. Hayne C, Xiang X and Luo Z: MEK inhibition and phosphorylation of serine 4 on B23 are two coincident events in mitosis. Biochem Biophys Res Commun 321: 675-680, 2004.

17. Laird AD, Morrison DK and Shalloway D: Characterization of Raf-1 activation in mitosis. J Biol Chem 274: 4430-4439, 1999.

18. Ziogas A, Lorenz IC, Moelling K and Radziwill G: Mitotic Raf-1 is stimulated independently of Ras and is active in the cytoplasm. J Biol Chem 273: 24108-24114, 1998.

19. Dangi S and Shapiro P: Cdc2-mediated inhibition of epidermal growth factor activation of the extracellular signal-regulated kinase pathway during mitosis. J Biol Chem 280: 24524-24531, 2005.

20. Liu L, Shi H, Chen X and Wang Z: Regulation of EGF-stimulated EGF receptor endocytosis during M phase. Traffic 12: 201-217, 2011

21. Margadant C, Cremers L, Sonnenberg A and Boonstra J: MAPK uncouples cell cycle progression from cell spreading and cytoskeletal organization in cycling cells. Cell Mol Life Sci 70 293-307, 2013
22. Shi $\mathrm{H}$ and Shen L: The alteration of extracellular signal-regulated kinase (ERK) activity of COS7 cells from interphase to mitosis. Cell Mol Biol (Noisy-le-grand) 56 Suppl: OL1427-OL1433, 2010.

23. Anderson D, Koch CA, Grey L, Ellis C, Moran MF and Pawson T: Binding of SH2 domains of phospholipase $\mathrm{C}$ gamma 1, GAP, and Src to activated growth factor receptors. Science 250: 979-982, 1990.

24. Moran MF, Koch CA, Anderson D, et al: Src homology region 2 domains direct protein-protein interactions in signal transduction. Proc Natl Acad Sci USA 87: 8622-8626, 1990.

25. Koch CA, Anderson D, Moran MF, Ellis C and Pawson T: SH2 and SH3 domains: elements that control interactions of cytoplasmic signaling proteins. Science 252: 668-674, 1991.

26. Pawson T: Protein modules and signalling networks. Nature 373 : 573-580, 1995

27. Zhao Y, Thomas HD, Batey MA, et al: Preclinical evaluation of a potent novel DNA-dependent protein kinase inhibitor NU7441. Cancer Res 66: 5354-5362, 2006.

28. Ma DM, Ji YJ, Yang F, Liu W, Wan Z and Li RY: Effects of U0126 on growth and activation of mitogen-activated protein kinases in Aspergillus fumigatus. Chin Med J (Engl) 126: 220-225, 2013

29. Newton R, Cambridge L, Hart LA, Stevens DA, Lindsay MA and Barnes PJ: The MAP kinase inhibitors, PD098059, UO126 and SB203580, inhibit IL-1beta-dependent PGE(2) release via mechanistically distinct processes. Br J Pharmacol 130: 1353-1361, 2000.

30. Nam HJ, Kim S, Lee MW, et al: The ERK-RSK1 activation by growth factors at G2 phase delays cell cycle progression and reduces mitotic aberrations. Cell Signal 20: 1349-1358, 2008.

31. Rao VN and Reddy ES: elk-1 proteins interact with MAP kinases. Oncogene 9: 1855-1860, 1994. 